\title{
Suppression of galectin-3 in KSHV infected DMVEC cells and Kaposi's sarcoma: implications for tumorigenesis DJ Alcendor*1, WQ Zhu' ${ }^{1}$, P Desai ${ }^{2}$ and GS Hayward ${ }^{2}$
}

Address: ${ }^{1}$ Meharry Medical College, School of Medicine, Center for AIDS Health Disparities Research and Department of Microbial Pathogenesis and Immune Response, Nashville, Tennessee, USA and '2Department of Viral Oncology and Pharmacology, Molecular Virology Laboratories, Sidney-Kimmel Cancer Research Center, Johns Hopkins University School of Medicine, Baltimore, Maryland, USA

* Corresponding author

from I I th International Conference on Malignancies in AIDS and Other Acquired Immunodeficiencies (ICMAOI): Basic, Epidemiologic, and Clinical Research

Bethesda, MD, USA. 6-7 October 2008

Published: 17 June 2009

Infectious Agents and Cancer 2009, 4(Suppl 2):P5 doi:I0.I I86/I750-9378-4-S2-P5

This abstract is available from: http://www.infectagentscancer.com/content/4/S2/P5

(c) 2009 Alcendor et al; licensee BioMed Central Ltd.

The Galectins are a family of proteins that share an affinity for Beta-galactoside containing glycoconjugates. Galectin3 has been implicated in tumor progression and metastasis. It is also known that in prostate, ovarian and breast cancer, down regulation of Galectin-3 is associated with malignancy. Kaposi's sarcoma (KS) is characterized as an angioproliferative tumor of vascular endothelial cells and produces rare $\mathrm{B}$ cell lymphoproliferative diseases in the form of primary effusion lymphomas (PEL) and some forms of Multicentric Castleman's Disease (MDC). Kaposi's Sarcoma-Associated Herpesvirus also known as KSHV or Human Herpesvirus type 8 (HHV8) is the etiological agent of KS. We have observed suppression of galectin-3 expression in Kaposi's sarcoma (KS). Here we demonstrate that galectin-3 protein expression is downregulated 20-fold in KSHV infected dermal microvascular endothelial cells (DMVEC) cells. We show loss of Galectin-3 staining by dual labeled immunofluorescence in latency associated nuclear antigen (LANA) positive spindle cells. We also find reduced levels of galectin-3 expression in LANA positive spindle cell regions in archival KS tissue. There is also transcriptional suppression of Galectin-3 message in KSHV infected DMVEC cells compared to mock infected controls. We demonstrate that KSHV vFLIP is the likely viral gene that targets galectin-3 downregulation in HeLa cells. In pleural effusion lymphoma cell lines (PEL), we observe different levels of galectin-3 expression associated with varying levels of KSHV replication. The search for novel host cell factors that may contribute to the overall pathogenesis of KS is essential for early detection of KS and development of innovative therapies for treatment. 